\title{
openheart Balloon-expandable transfemoral transcatheter aortic valve implantation with or without predilation: findings from the prospective EASE-IT TF multicentre registry
}

Gerhard Schymik, ${ }^{1}$ Tanja Rudolph, ${ }^{2}$ Claudius Jacobshagen, ${ }^{3}$ Jürgen Rothe, ${ }^{4}$ Hendrik Treede, ${ }^{5}$ Sebastian Kerber, ${ }^{6}$ Derk Frank (D) , ${ }^{7,8}$ Lenka Sykorova, ${ }^{9}$ Maki Okamoto, ${ }^{10}$ Martin Thoenes, ${ }^{11}$ Cornelia Deutsch, ${ }^{12}$ Peter Bramlage (D) , ${ }^{12}$ Christian Butter $^{10}$

To cite: Schymik G, Rudolph T, Jacobshagen C, et al. Balloonexpandable transfemoral transcatheter aortic valve implantation with or without predilation: findings from the prospective EASE-IT TF multicentre registry. Open Heart 2019;6:e001082. doi:10.1136/ openhrt-2019-001082

Received 4 May 2019 Revised 16 July 2019 Accepted 12 September 2019

D) Check for updates

(C) Author(s) (or their employer(s)) 2019. Re-use permitted under CC BY-NC. No commercial re-use. See rights and permissions. Published by BMJ.

For numbered affiliations see end of article.

\section{Correspondence to}

Dr Peter Bramlage, Institute for Pharmacology and Preventive Medicine, Cloppenburg, Germany; submission@ippmed. de

\section{ABSTRACT}

Background Predilation of the native valve has long been deemed necessary in transfemoral transcatheter aortic valve implantation (TF-TAVI), despite little trial evidence to support its clinical use. As most evidence is derived from retrospective analyses of observational studies, we conducted a two-armed, prospective multicentre registry. Methods Patients undergoing TF-TAVI with the Edwards SAPIEN 3 valve, with or without balloon aortic valvuloplasty (BAV), were included and their procedural characteristics, short-term safety and short-term efficacy outcomes compared. We hypothesised that BAV may be safely omitted in many patients and omission could be associated with procedural benefits.

Results Overall, 196 consecutive patients underwent TF-TAVI, 56 with BAV and 140 without. The mean age was $81.2 \pm 6.2$ years, and the mean logistic EuroSCORE I was 17.1 \pm 13.6 . Device success according to Valve Academic Research Consortium-2 (VARC-2) was achieved in $96.4 \%$. The median procedural duration was shorter without BAV (56 min vs $90 \mathrm{~min} ; p=0.001$ ), as was fluoroscopy time (10 min vs $13 \mathrm{~min} ; p=0.001)$. The need for balloon postdilation was less frequent in patients without BAV ( $15.7 \%$ vs $30.4 \%, p=0.029)$. There was no difference in the proportion of patients meeting the VARC-2 defined composite safety endpoint at 30 days $(9.3 \%$ without vs $8.9 \%$ with BAV; adjusted OR (adjOR) 2.55; 95\% Cl 0.56 to 18.84 ) and at 6 months (15.2\% without vs $16.4 \%$ with BAV; adjOR 1.66; 95\% Cl 0.49 to 6.55).

Conclusions In the majority of patients, BAV can be safely omitted from the TAVI procedure without adverse effects.

The omission of BAV is associated with shorter procedural duration and could be advantageous for the majority of patients.

Trial registration number NCT02760771.

\section{INTRODUCTION}

Conventionally, balloon aortic valvuloplasty (BAV) to dilate the native valve prior to prosthesis delivery has been perceived as an

\section{Key questions}

What is already known about this subject?

- Balloon aortic valvuloplasty (BAV) is used to be a common step in transcatheter aortic valve replacement (TAVI). Although it was felt to be a procedural requirement, it was associated with a number of potential adverse events.

What does this study add?

- Since the early days of TAVI, the situation changed in that more and more patients received a valve implant without BAV. This situation has been picked up by this two-armed registry in an attempt to outline the efficacy and safety of skipping BAV. Based on the data, we can conclude that BAV can be safely omitted from the TAVI procedure without adverse effects. On the contrary, omission adds safety and shortens the procedure.

How might this impact on clinical practice?

- While earlier on there was uncertainty in which patients we are able to omit BAV, it now has become common practice and it turned into a need for reasoning why BAV should be performed in specific cases.

essential step in transcatheter aortic valve implantation (TAVI). ${ }^{1}$ Reasons for its performance include the estimation of the required implantation depth, reduce balloon slipping, facilitate aortic annulus crossing and maximise prosthetic heart valve (transcatheter heart valve (THV)) expansion. ${ }^{1}$ However, it also requires rapid ventricular pacing and has been associated with several adverse effects, such as transient coronary, cerebral and renal ischaemia; haemodynamic instability; and provocation of systemic inflammatory response syndrome. ${ }^{2}$ Arrhythmias 
may also arise, placing the patient at risk of cardioembolic events and potentially mandating permanent pacemaker implantation (PPI). ${ }^{3}$ Furthermore, the additional manipulation of the valve and access vessels may result in increased dislodgement of embolic material, increasing the potential for ischaemic stroke. ${ }^{4}$ Therefore, TAVI is increasingly being performed without BAV. ${ }^{5}$

Clinical reports have suggested that TAVI without BAV is feasible and safe, with adequate procedural efficacy. ${ }^{16-11}$ Nevertheless, there is no prospective clinical study comparing the two treatment options in patients undergoing transfemoral Edwards SAPIEN 3 implantation. Therefore, EASE-IT TF was designed as a prospective, two-armed, controlled-cohort study in patients undergoing TF-TAVI using the Edwards SAPIEN 3 heart valve where patients were assigned to a procedure with or without predilation of the aortic valve. We hypothesised that BAV can be safely omitted in the majority of patients and omission being associated with procedural benefits.

\section{METHODS}

The design and rationale of EASE-IT TF have been previously described. ${ }^{12}{ }^{13}$ Patients undergoing TF-TAVI at 10 experienced sites were enrolled between May and November 2016 and the patient followed throughout 2017.

\section{Study participants and intervention}

Patients who were $\geq 18$ years of age at the time of enrolment and had an indication for TF-TAVI according to the Edwards SAPIEN 3 THV instructions for use were included. All patients underwent TAVI with the Edwards SAPIEN 3 THV via the TF access route. Performance or omission of aortic valve predilation was at the discretion of the treating physician and the decision to perform or omit BAV was documented at patient inclusion.

\section{Documentation and endpoints}

The primary composite safety endpoint was the rate of all-cause mortality, stroke, non-fatal myocardial infarction (MI), acute kidney injury (AKI) and PPI at 30 days, as defined in the Valve Academic Research Consortium-2 (VARC-2) consensus document. ${ }^{14}$ This set of criteria was reassessed again as a secondary safety endpoint at 6 months. Further secondary endpoints included the rate of each of the aforementioned individual events at 30 days and 6 months, as well as the change in New York Heart Association (NYHA) class at 30 days and 6 months, relative to baseline. Device success, periprocedural complications, procedural time, contrast agent volume and catecholamine use were also assessed. The treating physicians were also asked to indicate their reason(s) for performing/omitting BAV in each patient.

\section{Calcium assessment}

The methods for calcium assessment were described previously. ${ }^{13}$ In short, a routine multi-slice computed tomography (MSCT) scan performed prior to TAVI was obtained for each patient as per local practice. Contrast agent was used at all centres and calcium quantification was performed by a single designated, experienced core laboratory. For the assessment of valvular calcification, the aortic root was separated into three regions along its double-oblique long axis: the annulus (extending from $2 \mathrm{~mm}$ below to $3 \mathrm{~mm}$ above the annulus plane); the leaflet (extending from the annulus plane to the superior edge of the leaflets); and the LVOT (extending from the annulus plane to $5 \mathrm{~mm}$ immediately below it). These regions were further divided into three distinct sectors across the annular plane, corresponding to the non-coronary (NC), left coronary (LC) and right coronary (RC) cusps. For each of the nine resulting aortic root loci, the 3mensio Valves software (PIE Medical Imaging, Maastricht, the Netherlands ${ }^{15}$ ) was used to measure calcification (expressed in $\mathrm{mm}^{3}$ ), as previously described by Khalique et $\mathrm{al}^{16}$ with a 550 -HU threshold. Asymmetry was assessed by calculating the maximum absolute difference in calcification volume between any two leaflet sectors within each region.

\section{Statistical analysis}

Comparisons between TAVI patients with and without BAV were performed using Fisher's exact test for categorical variables and a t-test or Wilcoxon signed-rank test for continuous variables. Kaplan-Meier survival estimates were compared using a log-rank test. Potential bias introduced by differences in baseline characteristics between patients with or without BAV was adjusted for in a multivariate logistic regression analysis. Variables adjusted for included age, gender, prior MI, prior stroke/transient ischaemic attack (TIA), serum creatinine $\geq 2.0 \mathrm{mg} / \mathrm{dL}$, left ventricular ejection fraction and NYHA class. The results are expressed as OR with their 95\% CI. All statistical analysis was carried out using R (V.3.4.2 (2017-0928); https:/ /www.R-project.org/), with a p value of $<0.05$ considered statistically significant.

\section{RESULTS}

Of the 200 patients documented in the registry, 4 did not meet the eligibility criteria, resulting in an analysis population of 196 patients (figure 1). Physicians decided prior to the procedure to omit $\mathrm{BAV}$ in 146 patients and to perform BAV in 54 patients. In nine patients, the initial decision was reconsidered during the intervention, with six patients receiving unplanned BAV and three patients omitting planned BAV. This resulted in 140 patients undergoing the procedure without predilation of the aortic valve $(71.4 \%)$ and 56 patients receiving predilation $(28.6 \%)$.

\section{Patient characteristics}

Patients included had a mean age of 81.2 years, a logistic EuroSCORE I of $17.1 \%$ and a predominance of male (55.1\%; table 1). A high proportion of patients had coronary artery disease and/or had previously undergone one or more cardiovascular intervention. Comorbidities such 


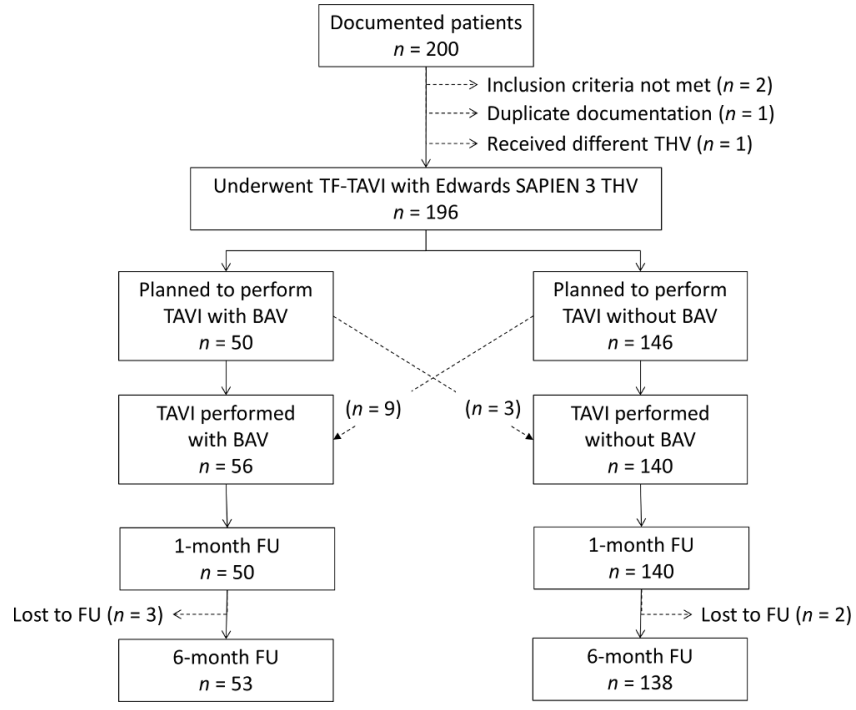

Figure 1 Patient flow. BAV, balloon aortic valvuloplasty; FU, follow-up; TF-TAVI, transfemoral transcatheter aortic valve implantation; THV, transcatheter heart valve.

as systemic hypertension, pulmonary hypertension and diabetes were common. The majority of patients were in NYHA class III/IV.

Baseline characteristics were similar between groups, with the only difference being hypertension $(78.6 \%$ in patients with vs $92.1 \%$ in patients without $\mathrm{BAV} ; \mathrm{p}=0.013$ ) and syncope/dizziness on exertion (21.4\% in patients with vs $36.4 \%$ inpatients without BAV; $\mathrm{p}=0.042$ ). There was a borderline-significant trend towards higher $\mathrm{V}_{\max }$ values in patients with BAV ( $p=0.065)$. Furthermore, the degree of calcification as determined based on CT scans was not different between groups with a particularly low burden of calcium in the left ventricular outflow tract.

\section{Reasons for planned performance/omission of BAV}

Treating physicians cited a desire to reduce the procedural duration in 109 out of 146 patients (74.7\%), followed by a perceived risk of cerebral microemboli $(64.1 \%)$ and the perceived risk of annulus rupture $(24.0 \%)$ and AV block $(22.1 \%)$ as principal reasons for omitting BAV (table 2). In the majority of patients $(31 / 50)$, assistance in crossing the aortic valve was the principal reason for performing BAV, followed by addressing doubts over the choice of valve size $(26.0 \%)$.

Reasons for omitting planned BAV $(n=3)$ was the perceived risk of annulus rupture $(n=1)$ and other not predefined reasons $(n=2)$. Important reasons to switch from planned omission to the performance of BAV $(n=9)$ were a desired assistance in crossing the aortic valve $(n=5)$ and assistance in choosing the correct valve size $(n=3)$.

\section{Procedural details and outcomes}

In the no BAV group, procedural duration and fluoroscopy time were both shorter (56 min vs $90 \mathrm{~min}$, $\mathrm{p}=0.001$ and $10.0 \mathrm{~min}$ vs $13.0 \mathrm{~min}, \mathrm{p}=0.001$, respectively), while there only was a statistically non-significant difference in the contrast volume used (table 3). Postdilation was performed in a significantly smaller proportion of patients without BAV compared with those with BAV ( $15.7 \%$ vs $30.4 \%$; $\mathrm{p}=0.029$ ).

VARC-2-defined device success was not statistically different in patients without compared with those with BAV (97.9\% vs $92.9 \%$; $\mathrm{p}=0.104)$. In six patients who experienced device failure, the reason for this was a mean AV gradient of $>20 \mathrm{~mm} \mathrm{Hg}$ post-TAVI. One further patient also required a second valve due to suboptimal device positioning (TAVI without BAV group). No patients died during the procedure.

There were no significant differences in the frequency of procedural complications between the two groups (table 3). Three patients experienced haemodynamic instability, of which two required catecholamine administration (one patient per group).

\section{Outcomes at $\mathbf{3 0}$ days and $\mathbf{6}$ months}

At 30 days post-TAVI, the primary safety composite endpoint was met by $9.4 \%$ of patients, mortality was $1.0 \%$, major bleeding complications was $7.2 \%$ and patients in NYHA III/IV 7.3\%. Comparable proportions of patients in the TAVI without BAV and TAVI with BAV groups met the primary safety composite endpoint (9.3\% vs $8.9 \%)$, with BAV not significantly influencing this outcome (adjusted OR 2.55; 95\% CI 0.56 to 18.84) (table 4). Similarly, performance/omission of BAV was not significantly associated with any of the individual components of this endpoint. No stroke occurred and the rates of renal failure and PPI were similar between groups. Death occurred in two patients without BAV (one fatal MI, one dissection) and no patient with BAV. BAV omission was associated with a clinically (if not statistically) significant reduction in the likelihood of major vascular complications (adjusted OR 0.20; 95\% CI 0.03 to 1.03). No patients were in Canadian Cardiovascular Society (CCS) angina class III/IV at 30 days, and NYHA class III/IV was low and comparable between groups $(8.1 \%$ vs $5.4 \%$; adjusted OR 3.02; $95 \%$ CI 0.52 to 28.35 ) (table 4 ).

At 6 months post-TAVI, the secondary safety composite endpoint was met by $15.5 \%$ of patients, mortality was $4.7 \%$ (figure 2), the rate of new pacemaker was $10.1 \%$ and, again, NYHA III/IV was 7.1\%. Comparable proportions of patients with or without BAV met the secondary safety composite endpoint ( $16.4 \%$ vs $15.2 \%)$, with BAV not significantly influencing this outcome (adjusted OR $1.66 ; 95 \%$ CI 0.49 to 6.55 ) (table 5 ).

\section{Patient and disease characteristics by BAV reasoning}

To further explore differences between patients where 'stretching of the aortic wall to crack calcifications' or 'assistance in crossing the aortic valve' were principal reasons for BAV performance, versus those with other reasons (assistance in choice of valve size, reduction of PVL), we found no systematic differences in patient characteristics or outcomes (online supplementary table 1). 
Table 1 Patient and disease characteristics at baseline

\begin{tabular}{|c|c|c|c|c|}
\hline & \multirow{2}{*}{$\begin{array}{l}\text { Total } \\
\begin{array}{l}\text { Mean } \pm \text { SD or } n / N ~(\%) \\
(n=196)\end{array}\end{array}$} & \multirow{2}{*}{$\begin{array}{l}\text { TAVI with BAV } \\
\text { Mean } \pm \text { SD or } n / N(\%) \\
(n=56)\end{array}$} & \multirow{2}{*}{$\begin{array}{l}\text { TAVI without BAV } \\
\text { Mean } \pm \text { SD or } n / N(\%) \\
(n=140)\end{array}$} & \multirow[b]{2}{*}{$P$ value } \\
\hline & & & & \\
\hline Age (years) & $81.2 \pm 6.2$ & $80.3 \pm 5.9$ & $81.5 \pm 6.3$ & $0.178^{*}$ \\
\hline Female gender & $88 / 196(44.9)$ & $23 / 56(41.1)$ & $65 / 140(46.4)$ & 0.528 \\
\hline Body mass index $\left(\mathrm{kg} / \mathrm{m}^{2}\right)$ & $27.3 \pm 4.8$ & $27.8 \pm 5.4$ & $27.1 \pm 4.5$ & 0.318 \\
\hline \multicolumn{5}{|l|}{ Non-cardiac comorbidities } \\
\hline Hypertension & 173/196 (88.3) & 44/56 (78.6) & $129 / 140(92.1)$ & 0.013 \\
\hline Diabetes & 62/194 (32.0) & 18/54 (33.3) & 44/140 (31.4) & 0.864 \\
\hline Stroke/TIA & 27/194 (13.9) & $5 / 56(8.9)$ & 22/138 (15.9) & 0.256 \\
\hline PAD & 22/195 (11.3) & $8 / 56(14.3)$ & $14 / 139(10.1)$ & 0.454 \\
\hline Pulmonary hypertension & 70/188 (37.2) & $16 / 52(30.8)$ & $54 / 136(39.7)$ & 0.257 \\
\hline Creatinine $\geq 2.0 \mathrm{mg} / \mathrm{dL}$ & 9/196 (4.6) & $3 / 56(5.4)$ & $6 / 140(4.3)$ & 0.717 \\
\hline Dialysis & 3/196 (1.5) & $1 / 56(1.8)$ & $2 / 140(1.4)$ & 1.000 \\
\hline \multicolumn{5}{|l|}{ Cardiac history } \\
\hline CAD & $129 / 196(65.8)$ & $37 / 56(66.1)$ & $92 / 140(65.7)$ & 1.000 \\
\hline Prior MI & 24/196 (12.2) & $6 / 56(10.7)$ & $18 / 140(12.9)$ & 0.812 \\
\hline Prior CV intervention & 78/196 (39.8) & $21 / 56(37.5)$ & $57 / 140(40.7)$ & 0.748 \\
\hline Prior pacemaker/ICD & 22/196 (11.2) & $7 / 56(12.5)$ & $15 / 140(10.7)$ & 0.803 \\
\hline Logistic EuroSCORE I (\%) & $17.1 \pm 13.6$ & $16.1 \pm 12.1$ & $17.5 \pm 14.1$ & $0.706^{*}$ \\
\hline \multicolumn{5}{|l|}{ Echocardiographic parameters } \\
\hline $\mathrm{EOA}\left(\mathrm{cm}^{2}\right)$ & $0.73 \pm 0.18$ & $0.69 \pm 0.17$ & $0.75 \pm 0.19$ & 0.094 \\
\hline Peak AV gradient (mm Hg) & $70.9 \pm 22.4$ & $74.9 \pm 21.7$ & $69.0 \pm 22.5$ & 0.463 \\
\hline Mean AV gradient (mm Hg) & $44.1 \pm 15.3$ & $47.2 \pm 15.7$ & $42.8 \pm 14.9$ & $0.138^{*}$ \\
\hline $\mathrm{V}_{\max }(\mathrm{m} / \mathrm{s})$ & $4.2 \pm 0.8$ & $4.4 \pm 0.6$ & $4.1 \pm 0.8$ & $0.065^{\star}$ \\
\hline LVEF (\%) & $52.5 \pm 12.4$ & $53.2 \pm 11.9$ & $52.1 \pm 12.6$ & $0.369^{*}$ \\
\hline NYHA class III/IV & 138/191 (72.3) & $38 / 55(69.1)$ & $100 / 136(73.5)$ & 0.593 \\
\hline CCS angina class III/IV & 27/187 (14.4) & 6/51 (11.8) & $21 / 136(15.4)$ & 0.644 \\
\hline Syncope/dizziness on exertion & $63 / 196(32.1)$ & $12 / 56(21.4)$ & $51 / 140(36.4)$ & 0.042 \\
\hline Calcification & $\begin{array}{l}\text { Median (IQR) } \\
\mathrm{n}=178\end{array}$ & $\begin{array}{l}\text { Median (IQR) } \\
\mathrm{n}=55\end{array}$ & $\begin{array}{l}\text { Median (IQR) } \\
\mathrm{n}=123\end{array}$ & $P$ value \\
\hline AnnulusCa $\left(\mathrm{mm}^{3}\right)$ & $17.3(2.7-50.3)$ & $13.2(2.4-49.6)$ & $18.7(3.1-53.1)$ & 0.773 \\
\hline LeafletCa $\left(\mathrm{mm}^{3}\right)$ & $272.1(137.1-484.3)$ & $317.5(99.7-647.0)$ & $269.3(150.7-435.1)$ & 0.636 \\
\hline LVOTCa $\left(\mathrm{mm}^{3}\right)$ & $0.0(0.0-6.8)$ & $0.0(0.0-4.9)$ & $0.0(0.0-7.3)$ & 0.735 \\
\hline Bicuspid valve & $2 / 177(1.1)$ & $1 / 55(1.8)$ & $1 / 124(0.8)$ & 0.521 \\
\hline
\end{tabular}

AnnulusCa: volume of calcification between $2 \mathrm{~mm}$ below and $3 \mathrm{~mm}$ above the annulus plane.

LeafletCa: volume of calcification between the annulus plane and superior edge of the leaflets.

LVOTCa: volume of calcification within the $5 \mathrm{~mm}$ immediately below the annulus plane.

*Wilcoxon test.

$\mathrm{AV}$, aortic valve; AVA, aortic valve area; BAV, balloon aortic valvuloplasty; CAD, coronary arterydisease; CCS, Canadian Cardiovascular Society; CV, cardiovascular; ICD, implantable cardioverter defibrillator; LVEF, left ventricular ejection fraction; MI, myocardial infraction; NYHA, New York HeartAssociation; PAD, peripheral artery disease; TAVI, transcatheter aortic valve implantation; TIA, transient ischaemic attack; $\mathrm{V}_{\max }$, maximum velocity.

\section{DISCUSSION}

In the present real-world analysis of patients undergoing TF-TAVI with the Edwards SAPIEN 3 THV, omission/ performance of BAV did not significantly influence VARC-2-defined early safety, nor the individual rates of stroke, AKI, PPI or mortality up to 6 months. Nevertheless, procedural and fluoroscopy times were significantly shorter when BAV was not performed. Accordingly, the most common reason given by physicians for not performing BAV was to save time, with perceived risk of microemboli also playing a role in this decision. Our data suggest that BAV may be omitted in many patients 
Table 2 Reasons for planned omission/performance of $\mathrm{BAV}^{*}$

$\mathrm{n} / \mathrm{N}(\%)$

\begin{tabular}{|ll}
\hline Reasons given for BAV omission ( $\mathrm{n}=146)$ & \\
\hline Desire to shorten procedural duration & $109 / 146(74.7)$ \\
\hline Cost reduction & $1 / 145(0.7)$ \\
\hline Avoidance of rapid pacing & $16 / 145(11.0)$ \\
\hline Perceived risk of annulus rupture & $35 / 146(24.0)$ \\
\hline Perceived risk of cerebral microemboli & $93 / 145(64.1)$ \\
\hline Risk of AV block & $32 / 145(22.1)$ \\
\hline Other reason & $7 / 145(4.8)$ \\
\hline Reasons given for BAV performance (n=50) & \\
\hline Rehearsal before final positioning/release & $0 / 50(0)$ \\
\hline Assistance in choice of valve size & $13 / 50(26.0)$ \\
\hline $\begin{array}{l}\text { Stretching of the aortic wall to crack } \\
\text { calcifications }\end{array}$ & $5 / 50(10.0)$ \\
\hline $\begin{array}{l}\text { Assistance in crossing the aortic valve } \\
\text { Reduction of PVL }\end{array}$ & $31 / 50(62.0)$ \\
\hline $\begin{array}{l}\text { Reduction of radial force needed for valve } \\
\text { expansion }\end{array}$ & $2 / 50(4.0)$ \\
\hline Other reason & $0 / 50(0)$ \\
\hline
\end{tabular}

*More than one reason may apply per patient.

$\mathrm{AV}$, atrioventricular;BAV, balloon aortic valvuloplasty; PVL, paravalvular leak; patient. TAVI, transcatheter aortic valve implantation.

undergoing TF-TAVI and may be performed more often in clinical practice than indicated.

\section{BAV and procedural characteristics}

Multiple TF-TAVI studies have found procedural durations to be significantly shorter in the absence of predilation, with reported times of 50.5-133.7 min where BAV is performed compared with $34.0-108.7 \mathrm{~min}$ when it is not. ${ }^{6717-20}$ Concurrently, omission of BAV in the present study resulted in procedural times that were an average of $34 \mathrm{~min}$ shorter, as well as reduced fluoroscopy times, confirming its time-saving advantage. There was a trend for a reduced volume of contrast use, which was statistically not significant. The increasing importance of such parameters in clinical practice is underlined by physicians citing time concerns as a motive for omitting BAV in over $70 \%$ of the respective patients. Surprisingly, there was an increased requirement for balloon postdilation in patients with prior BAV (30.4\% vs $15.7 \%)$. Principal reasons for postdilation are residual paravalvular leaks (no difference in the current dataset) or residual gradients after the implantation $(7.1 \%$ vs $1.4 \%$ had a mean AV gradient $>20 \mathrm{~mm} \mathrm{Hg}$ ). This may be triggered by calcification or an underexpansion of the valve.

\section{BAV and procedural outcomes}

In the present study, device success was nominally more common in patients without BAV. This difference was principally driven by a lower proportion of patients having a postprocedural mean AV gradient $>20 \mathrm{~mm} \mathrm{Hg}$. It has been suggested that such gradients indicate a THV stenosis. We explored this in more detail according to the valve size selected and the degree of under-, normaland oversizing in the two different groups, but rates of these two parameters were not significantly different (see table 2). A potential, but not recorded reason for a postinterventional THV stenosis is the underfilling of the balloon during implantation. This matters as the valves have a nominal diameter, which is clearly affected by the volume used for inflating the balloon. A less than nominal filling volume would result in a smaller than nominal valve area.

As a consequence of rapid ventricular pacing, BAV and/or valve deployment, patients may experience bradycardia, AV block, coronary obstruction and/or severe aortic regurgitation. All of these conditions may require treatment with catecholamines. In the present study, the number of patients requiring catecholamine treatment for low cardiac output was extremely low (1.0\% overall). This is in contrast to the findings of the EASE-IT transapical (TA) registry analysis, in which $22.2 \%$ of patients undergoing TAVI via the TA route required catecholamines. ${ }^{21}$ One explanation for this difference may be the higher degree of cardiac manipulation during TA-TAVI, resulting in a greater potential for functional disturbances and reduced cardiac output. Indeed, inclusion of the BAV step exacerbated this effect in the TA cohort, with almost twice as many BAV patients receiving catecholamines compared with no BAV patients $(32.5 \%$ vs $17.5 \% ; \mathrm{p}=0.017) .{ }^{21}$ While catecholamine use was statistically comparable between groups in the present analysis, this may be the result of a low event rate masking statistical significance. Interestingly, catecholamine use has not been reported by the majority of TF-TAVI and TA-TAVI analyses. $136910171922-25$

\section{Effect of BAV on early and midterm outcomes}

BAV performance/omission had no significant effect on the likelihood of meeting the VARC-2 early safety composite endpoint. The rates observed in the present study were substantially lower than those reported by previous TF-TAVI studies, likely thanks to refinement of the TAVI technique and improved THV/delivery system design. ${ }^{6}{ }^{10}$ Nevertheless, the redundancy of BAV in relation to this outcome is consistent throughout studies, ${ }^{6} 101926$ with a meta-analysis reporting a non-significant trend towards a reduced risk of meeting the VARC-2 early safety endpoint when BAV is omitted (relative risk (RR) $0.68 ; 95 \%$ CI 0.44 to 1.07$).^{27}$

Mortality rates were low and similar between groups at 30 days, being at the lower end of the reported range for TF-TAVI patients without BAV (2.5\%-7.7\%). ${ }^{6} 1719222326$ The lack of prognostic value of BAV for mortality was further seen at 6 months, where survival was comparable between groups, although superior to the rates commonly reported for real-world Edwards SAPIEN 
Table 3 Procedural variables and outcomes of TF-TAVI

\begin{tabular}{llll}
$\begin{array}{l}\text { Total } \\
(n=196)\end{array}$ & $\begin{array}{l}\text { TAVI with BAV } \\
(n=56)\end{array}$ & $\begin{array}{l}\text { TAVI without BAV } \\
(n=140)\end{array}$ \\
\cline { 1 - 2 } $\begin{array}{l}n / N(\%) \text { or Median } \\
(\text { IQR })\end{array}$ & $\begin{array}{l}n / N(\%) \text { or Median } \\
(\text { IQR })\end{array}$ & $\begin{array}{l}n / N(\%) \text { or Median } \\
(\text { IQR })\end{array}$ \\
\hline
\end{tabular}

(IQR)

(IQR)

(IQR)

$P$ value

Anaesthesia

$149 / 196(76.0)$

General anaesthesia

47/196 (24.0)

47/56 (83.9)

0.101

Conscious sedation

9/56 (16.1)

02/140 (72.9)

Size of balloon used for predilation

$\begin{array}{lll}20 \mathrm{~mm} & 16 / 196(8.2) & 16 / 56(28.6) \\ 22 / 23 \mathrm{~mm} & 17 / 196(8.7) & 17 / 56(30.4) \\ 24 \mathrm{~mm} & 2 / 196(1.0) & 2 / 56(3.6) \\ 26 \mathrm{~mm} & 21 / 196(10.7) & 21 / 56(37.5)\end{array}$

Size of implanted THV

$1 / 196(0.5)$

$1 / 56(1.8)$

0.316

$20 \mathrm{~mm}$

$64 / 196(32.7)$

20/56 (35.7)

$0 / 140(0)$

$23 \mathrm{~mm}$

68/196 (34.7)

$13 / 56$ (23.2)

$44 / 140(31.4)$

$26 \mathrm{~mm}$

$63 / 196$ (32.1)

$22 / 56(39.3)$

$55 / 140$ (39.3)

$29 \mathrm{~mm}$

$14 / 179(7.8)$

$41 / 140(29.3)$

Sizing vs native annulus

\begin{tabular}{|c|c|c|c|c|}
\hline Undersized & $14 / 179(7.8)$ & $3 / 55(5.5)$ & $11 / 124(8.9)$ & \\
\hline Normal size & $121 / 179(67.6)$ & $38 / 55(69.1)$ & $83 / 124(66.9)$ & \\
\hline Oversized & $44 / 179(24.6)$ & $14 / 55(25.5)$ & $30 / 124(24.2)$ & \\
\hline Requirement for balloon postdilation & $39 / 196(19.9)$ & $17 / 56(30.4)$ & $22 / 140(15.7)$ & 0.029 \\
\hline Procedural duration (min) & $65(47 / 104)$ & $90(59 / 118)$ & $56(40 / 87)$ & 0.001 \\
\hline Fluoroscopy time (min) & $10.5(8.0 / 14.0)$ & $13.0(10.0 / 16.0)$ & $10.0(7.0 / 12.0)$ & 0.001 \\
\hline Contrast volume (ml) & $125(100 / 175)$ & $131(116 / 164)$ & $120(98 / 190)$ & 0.240 \\
\hline Device success ${ }^{\star} \dagger$ & $189 / 196(96.4)$ & $52 / 56(92.9)$ & $137 / 140(97.9)$ & 0.104 \\
\hline Absence of death & $196 / 196(100)$ & $56 / 56(100)$ & $140 / 140(100)$ & n.a. \\
\hline Correct positioning & 195/196 (99.5) & $56 / 56(100)$ & $139 / 140(99.3)$ & 1.000 \\
\hline Post-TAVI mean AV gradient $\leq 20 \mathrm{~mm} \mathrm{Hg}$ & $190 / 196(96.9)$ & $52 / 56(92.9)$ & $138 / 140(98.6)$ & 0.057 \\
\hline Paravalvular leakage & & & & 0.740 \\
\hline Mild & 26/196 (13.3) & $8 / 56(14.3)$ & $18 / 140(12.9)$ & \\
\hline Moderate/severe & $1 / 196(0.5)$ & $0 / 56(0)$ & $1 / 140(0.7)$ & \\
\hline Mean AV gradient $(\mathrm{mm} \mathrm{Hg})$ & $11.8 \pm 4.7$ & $12.9 \pm 5.3$ & $11.4 \pm 4.5$ & 0.058 \\
\hline \multicolumn{5}{|l|}{ Procedural complications } \\
\hline Access complications $\ddagger$ & 7/196 (3.6) & $0 / 56(0)$ & $7 / 140(5.0)$ & 0.195 \\
\hline Aortic root rupture & 0/196 (0) & $0 / 56(0)$ & $0 / 140(0)$ & n.a. \\
\hline Haemodynamic instability & 3/196 (1.5) & 2/56 (3.6) & $1 / 140(0.7)$ & 0.197 \\
\hline Catecholamine use & 2/196 (1.0) & $1 / 56(1.8)$ & $1 / 140(0.7)$ & 0.491 \\
\hline Complete AV block with PPI & $5 / 196(2.6)$ & 2/56 (3.6) & $3 / 140(2.1)$ & 0.625 \\
\hline Conversion to open surgery & 0/196 (0) & $0 / 56(0)$ & $0 / 140(0)$ & n.a. \\
\hline Device malfunction & 0/196 (0) & $0 / 56(0)$ & $0 / 140(0)$ & n.a. \\
\hline Need for second valve & $1 / 196(0.5)$ & $0 / 56(0)$ & $1 / 140(0.7)$ & 1.000 \\
\hline
\end{tabular}

${ }^{*}$ Defined according to VARC-2 as the absence of death, correct positioning of a single prosthetic heart valve and its intended performance. ${ }^{14}$ †Multiple reasons may apply.

¥Defined according to VARC-2 as dissection, aortic root rupture or uncontrolled bleeding. ${ }^{14}$

AV, aortic valve; BAV, balloon aortic valvuloplasty; n.a., not applicable; PPI, permanent pacemaker implantation; TAVI, transcatheter aortic valve implantation; THV, transcatheter heart valve; VARC-2, Valve Academic Research Consortium-2. 
Table 4 Effect of BAV omission on outcomes at 30 days

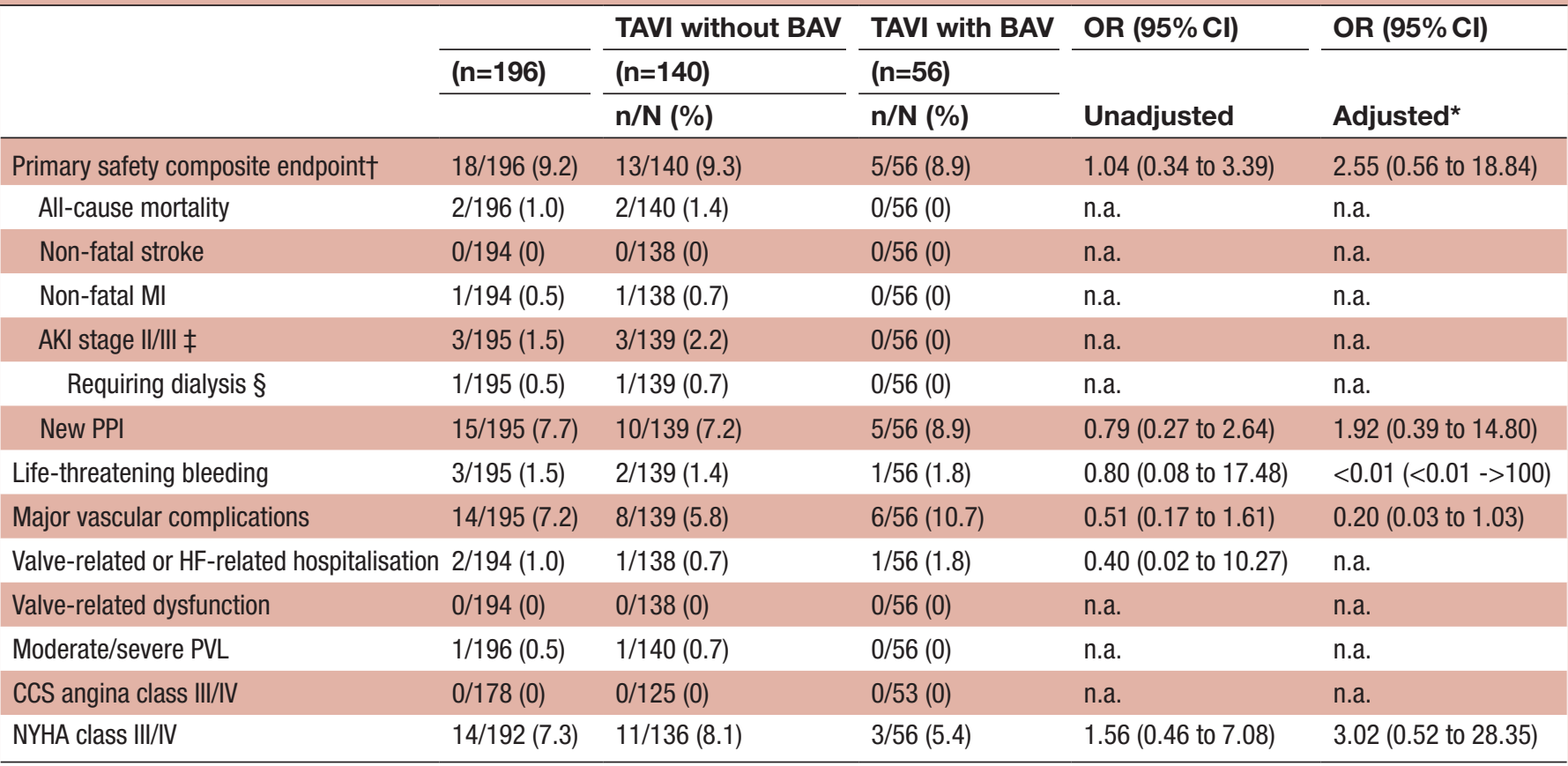

*Adjusted for baseline characteristics listed in table 1, including age, gender, prior myocardial infarction, prior stroke/transient ischaemic attack, serum creatinine $\geq 2.0 \mathrm{mg} / \mathrm{dL}$, left ventricular ejection fraction and NYHA class.

†Defined according to Valve Academic Research Consortium-2 as a composite of all-cause mortality, non-fatal stroke, non-fatal myocardial infarction, acute kidney injury and permanent pacemaker implantation. ${ }^{14}$

$\ddagger$ According to AKIN criteria.

§Excluding patients with dialysis prior to TAVI.

AKI, acute kidney injury; BAV, balloon aortic valvuloplasty; CCS, Canadian Cardiovascular Society; HF, heart failure; MI, myocardial infarction; NYHA, New York Heart Association; PPI, permanent pacemaker implantation; TAVI, transcatheter aortic valve implantation.

recipients (approximately 85\%). ${ }^{24}{ }^{25}$ The lack of influence of BAV on death has also been reported by an analysis of the observational UK TAVI registry (OR 1.04; $95 \%$ CI 0.63 to 1.72 ), supporting the present findings. ${ }^{5}$ However, a meta-analysis of several small TF-TAVI studies suggests a greater risk of death after TF-TAVI when BAV is performed. ${ }^{27}$ The influence of study design/setting may underlie this discrepancy.

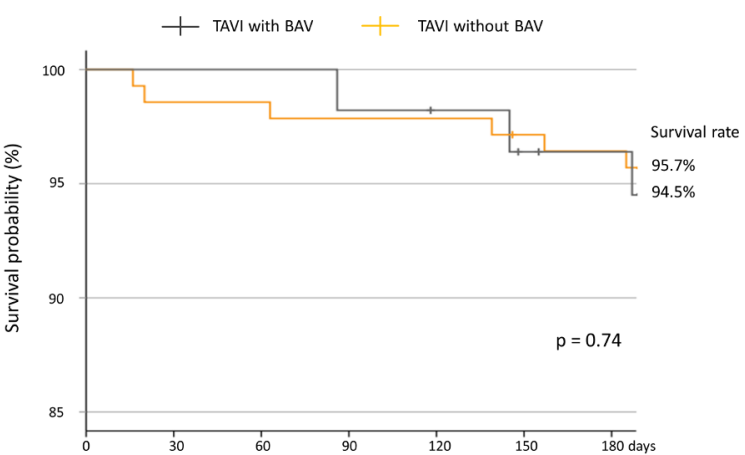

\begin{tabular}{lccccccc} 
Patients at risk & & & & & & \\
\hline TAVI with BAV & 56 & 56 & 56 & 55 & 54 & 52 & 51 \\
TAVI without BAV & 140 & 138 & 138 & 137 & 137 & 134 & 133 \\
\hline
\end{tabular}

Figure 2 Kaplan-Meier curves for survival over the 6 months post-TAVI. Between-group differences were tested using a log-rank test. BAV, balloon aortic valvuloplasty; TAVI, transcatheter aortic valve implantation.
Reportedly, $13 \%-25 \%$ of patients undergoing TAVI will require PPI due to conduction disturbances, leading to longer and more frequent hospitalisations and an increased risk of mortality. ${ }^{3}$ There is much controversy over whether or not BAV is independently associated with PPI. ${ }^{13568171926}$ In the present study, correction for confounders resulted in ORs for PPI at 30 days and 6 months that had extremely wide CIs, meaning that our data do not support the existence of a significant association. While the UK TAVI registry found omission of BAV to be predictive for PPI at univariate analysis (OR 1.30; $95 \%$ CI 0.99 to 1.96), this effect disappeared after multivariate adjustment. Bagur et al also reported no difference between TAVI with BAV and TAVI without BAV regarding the need for PPI (RR 0.79; 95\% CI 0.29 to 2.17). Thus, our data are consistent with some other studies that suggest BAV has no tangible effect on PPI rates.

\section{Potential limitations of EASE-IT TF}

First, the non-interventional registry design precluded randomisation, with physicians choosing particular patients in whom to perform or omit BAV. Consequently, a degree of selection bias was unavoidable; however, adjustment for confounders at multivariate analysis was intended to account for such baseline differences between groups, and the data collected allow a rare 
Table 5 Effect of BAV omission on outcomes at 6 months

\begin{tabular}{|c|c|c|c|c|c|}
\hline & Total & TAVI without BAV & TAVI with BAV & OR $(95 \% \mathrm{Cl})$ & OR $(95 \% \mathrm{Cl})$ \\
\hline & $(n=196)$ & $(n=140)$ & $(n=56)$ & \multirow[b]{2}{*}{ Unadjusted } & \multirow[b]{2}{*}{ Adjusted* } \\
\hline & $\mathrm{n} / \mathrm{N}(\%)$ & n/N (\%) & $\mathrm{n} / \mathrm{N}(\%)$ & & \\
\hline Secondary safety composite endpoint $†$ & $30 / 193(15.5)$ & 21/138 (15.2) & $9 / 55(16.4)$ & $0.92(0.40$ to 2.24$)$ & 1.66 (0.49 to 6.55$)$ \\
\hline All-cause mortality & 9/191 (4.7) & $6 / 138(4.4)$ & $3 / 53(5.7)$ & $0.76(0.19$ to 3.70$)$ & $1.81(0.03->100)$ \\
\hline Cardiac & 2/191 (1.0) & $1 / 138(0.7)$ & $1 / 53(1.9)$ & - & - \\
\hline Non-cardiac & 3/191 (1.6) & $3 / 138(2.2)$ & $0 / 53(0)$ & - & - \\
\hline Unknown & $4 / 191(2.1)$ & 2/138 (1.4) & 2/53 (3.8) & - & . \\
\hline Non-fatal stroke & 2/184 (1.1) & 2/134 (1.5) & $0 / 50(0)$ & n.a. & n.a. \\
\hline Non-fatal MI & $1 / 184(0.5)$ & $1 / 134(0.7)$ & $0 / 50(0)$ & n.a. & n.a. \\
\hline New PPI & 19/188 (10.1) & 12/135 (8.9) & $7 / 53(13.2)$ & $0.64(0.24$ to 1.82$)$ & 1.60 (0.39 to 8.43) \\
\hline Valve thrombosis requiring medication & 2/185 (1.1) & 2/134 (1.5) & $0 / 51(0)$ & n.a. & n.a. \\
\hline Endocarditis & $1 / 185(0.5)$ & $0 / 134(0)$ & $1 / 51(2.0)$ & n.a. & n.a. \\
\hline Valve-related or HF-related hospitalisation & $8 / 184(4.3)$ & $5 / 134(3.7)$ & $3 / 50(6.0)$ & $0.61(0.14$ to 3.05$)$ & 0.14 (0.01 to 1.22$)$ \\
\hline NYHA class III/IV & 13/183 (7.1) & $11 / 134(8.2)$ & $2 / 49(4.1)$ & 2.10 (0.54 to 13.91$)$ & $1.24(0.21$ to 11.17$)$ \\
\hline
\end{tabular}

*Adjusted for baseline characteristics listed in table 1, including age, gender, prior myocardial infarction, prior stroke/transient ischaemic attack, serum creatinine $\geq 2.0 \mathrm{mg} / \mathrm{dL}$, left ventricular ejection fraction, and NYHA class.

†Defined as per the Valve Academic Research Consortium-2 early safety composite (combined rate of all-cause mortality, non-fatal stroke, non-fatal myocardial infarction, acute kidney injury and/or permanent pacemaker implantation). ${ }^{14}$

BAV, balloon aortic valvuloplasty; CCS, Canadian Cardiovascular Society; HF, heart failure; MI, myocardial infarction; NYHA, New York Heart Association; PPI, permanent pacemaker implantation; TAVI, transcatheter aortic valve implantation.

insight into the motives behind patient selection for BAV. Second, 30-day and 6-month event rates were extremely low. While this means that patients were treated expertly, with extremely good outcomes, its combination with a modest sample size means that we may have lacked the necessary statistical power to detect small between-group differences.

\section{CONCLUSIONS}

The findings from the present EASE-IT TF registry analysis suggest that BAV predilation provides no apparent clinical advantage at 6 months in the majority of high-risk aortic stenosis (AS) patients undergoing TF-TAVI with the Edwards SAPIEN 3 THV.

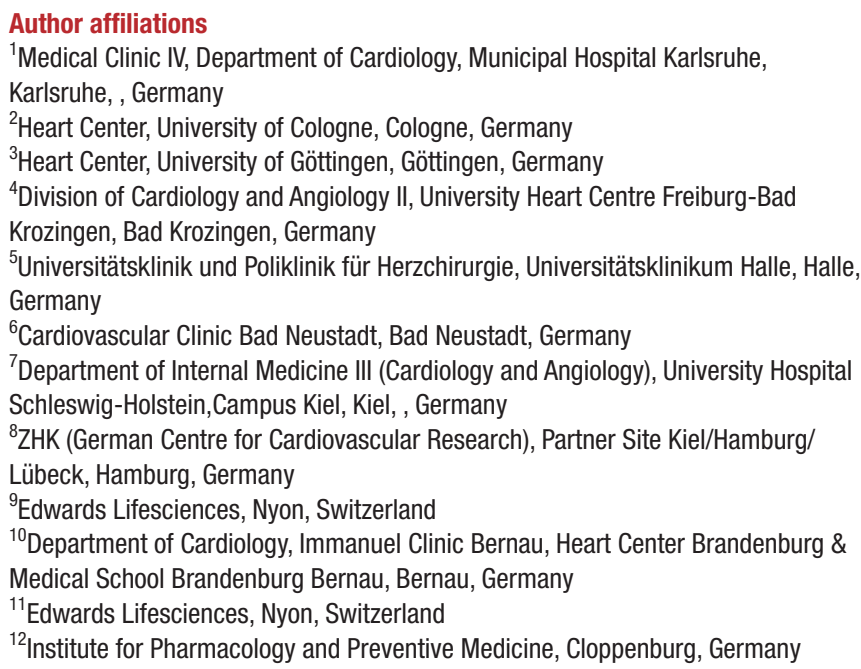

Acknowledgements Data are captured using the s4trials Software provided by Software for Trials Europe GmbH, Berlin, Germany. Expert statistical advice was provided by Dr Andreas Busjahn, HealthTwist GmbH, Berlin, Germany. Editorial support was provided by Helen Sims (Institute for Pharmacology and Preventive Medicine).

Contributors GS, PB, LS, MT and CB were involved in the conception and design of the study. The remaining authors gave feedback on the final protocol and enrolled patients (TKR, CJ, JR, HT, SK, DF), acquired further data (MO) or designed the analysis (GS, PB, CD). All authors (GS, TKR, CJ, JR, HT, SK, DF, LS, MO, MT, $\mathrm{CD}, \mathrm{PB}, \mathrm{CB}$ ) agreed on an interpretation of the data. GS, CD and PB drafted the manuscript and all other authors (TKR, CJ, JR, HT, SK, DF, LS, MO, MT, CB) revised the article for important intellectual content. All authors (GS, TKR, CJ, JR, HT, SK, DF, LS, M0, MT, CD, PB, CB) have given final approval of the version submitted. All authors (GS, TKR, CJ, JR, HT, SK, DF, LS, MO, MT, CD, PB, CB) are fully accountable for the content of the manuscript.

Funding Unrestricted educational research grant provided by Edwards Lifescience (Nyon, Switzerland) to the Institute for Pharmacology and Preventive Medicine (Cloppenburg, Germany).

Competing interests $\mathrm{PB}$ is the representative of the Institute for Pharmacology and Preventive Medicine, Cloppenburg (IPPMed), Germany. A research grant was provided by Edwards Lifescience, Nyon, Switzerland, to the Sponsor IPPMed. $\mathrm{CB}, \mathrm{TR}, \mathrm{CJ}, \mathrm{JR}, \mathrm{HT}, \mathrm{SK}, \mathrm{DF}$ and GS have received consultancy fees from different companies producing heart valves. LS and MTs are employees of Edwards Lifesciences.

Patient consent for publication Not required.

Ethics approval Ethical approval was obtained in writing from the appropriate ethics committees prior to patient enrolment and the study was conducted in accordance with the Declaration of Helsinki. All patients provided their written informed consent to participate.

Provenance and peer review Not commissioned; externally peer reviewed. Data availability statement Data are available on reasonable request.

Open access This is an open access article distributed in accordance with the Creative Commons Attribution Non Commercial (CC BY-NC 4.0) license, which permits others to distribute, remix, adapt, build upon this work non-commercially, and license their derivative works on different terms, provided the original work is 
properly cited, appropriate credit is given, any changes made indicated, and the use is non-commercial. See: http://creativecommons.org/licenses/by-nc/4.0/.

ORCID iDs

Derk Frank http://orcid.org/0000-0001-7561-075X

Peter Bramlage http://orcid.org/0000-0003-4970-2110

\section{REFERENCES}

1. Grube $E$, Naber C, Abizaid A, et al. Feasibility of transcatheter aortic valve implantation without balloon pre-dilation: a pilot study. JACC Cardiovasc Interv 2011;4:751-7.

2. Walther T, Dewey T, Borger MA, et al. Transapical aortic valve implantation: step by step. Ann Thorac Surg 2009;87:276-83.

3. Gensas CS, Caixeta A, Siqueira D, et al. Predictors of permanent pacemaker requirement after transcatheter aortic valve implantation: insights from a Brazilian registry. Int J Cardiol 2014;175:248-52.

4. Drews T, Pasic M, Buz S, et al. Transcranial Doppler sound detection of cerebral microembolism during transapical aortic valve implantation. Thorac Cardiovasc Surg 2011;59:237-42.

5. Martin GP, Sperrin M, Bagur R, et al. Pre-Implantation balloon aortic valvuloplasty and clinical outcomes following transcatheter aortic valve implantation: a propensity score analysis of the UK registry. $J$ Am Heart Assoc 2017;6.

6. Conradi L, Schaefer A, Seiffert M, et al. Transfemoral TAVI without pre-dilatation using balloon-expandable devices: a case-matched analysis. Clin Res Cardiol 2015;104:735-42.

7. Kim WK, Praz F, Blumenstein J, et al. Transfemoral aortic valve implantation of Edwards SAPIEN 3 without predilatation. Catheter Cardiovasc Interv 2016.

8. Fiorina C, Maffeo D, Curello S, et al. Direct transcatheter aortic valve implantation with self-expandable bioprosthesis: feasibility and safety. Cardiovasc Revasc Med 2014;15:200-3.

9. Möllmann H, Kim W-K, Kempfert J, et al. Transfemoral aortic valve implantation of Edwards SAPIEN XT without predilatation is feasible. Clin Cardiol 2014;37:667-71.

10. Aggarwal SK, Delahunty N, Wong B, et al. Balloon-Expandable transcatheter aortic valves can be successfully and safely implanted Transfemorally without balloon valvuloplasty. J Interv Cardiol 2016;29:319-24.

11. Bonaros N, Kofler M, Frank D, et al. Balloon-expandable transaortic transcatheter aortic valve implantation with or without predilation. $J$ Thorac Cardiovasc Surg 2018;155:915-23.

12. Butter C, Bramlage P, Rudolph T, et al. Balloon expandable transcatheter aortic valve implantation via the transfemoral route with or without pre-dilation of the aortic valve - rationale and design of a multicentre registry (EASE-IT TF). BMC Cardiovasc Disord 2016;16:223.

13. Butter C, Okamoto M, Schymik G, et al. Degree of valve calcification in patients undergoing Transfemoral transcatheter aortic valve implantation with and without balloon aortic valvuloplasty: findings from the multicenter EASE-IT TF registry. Catheter Cardiovasc Interv 2019.

14. Kappetein AP, Head SJ, Généreux $P$, et al. Updated standardized endpoint definitions for transcatheter aortic valve implantation: the valve academic research Consortium-2 consensus document (VARC-2). Eur J Cardiothorac Surg 2012;42:S45-S60.

15. Delgado V, Ng ACT, Schuijf JD, et al. Automated assessment of the aortic root dimensions with multidetector row computed tomography. Ann Thorac Surg 2011;91:716-23.

16. Khalique OK, Hahn RT, Gada H, et al. Quantity and location of aortic valve complex calcification predicts severity and location of paravalvular regurgitation and frequency of post-dilation after balloon-expandable transcatheter aortic valve replacement. JACC Cardiovasc Interv 2014;7:885-94.

17. Islas F, Almería C, García-Fernández E, et al. Usefulness of echocardiographic criteria for transcatheter aortic valve implantation without balloon predilation: a single-center experience. J Am Soc Echocardiogr 2015;28:423-9.

18. Bijuklic K, Haselbach T, Witt J, et al. Increased risk of cerebral embolization after implantation of a Balloon-Expandable aortic valve without prior balloon valvuloplasty. JACC Cardiovasc Interv 2015;8:1608-13.

19. Dumonteil N, Terkelsen C, Frerker C, et al. Outcomes of transcatheter aortic valve replacement without predilation of the aortic valve: insights from 1544 patients included in the source 3 registry. Int J Cardiol 2019. doi:10.1016/j.ijcard.2019.06.013. [Epub ahead of print: 12 Jun 2019].

20. Deharo P, Jaussaud N, Grisoli D, et al. Impact of Direct Transcatheter Aortic Valve Replacement Without Balloon Aortic Valvuloplasty on Procedural and Clinical Outcomes: Insights From the FRANCE TAVI Registry. JACC Cardiovasc Interv 2018;11:1956-65.

21. Strauch J, Wendt D, Diegeler A, et al. Balloon-expandable transapical transcatheter aortic valve implantation with or without predilation of the aortic valve: results of a multicentre registry. Eur $J$ Cardiothorac Surg 2017

22. Ferrera C, Nombela-Franco L, Garcia E, et al. Clinical and hemodynamic results after direct transcatheter aortic valve replacement versus pre-implantation balloon aortic valvuloplasty: a case-matched analysis. Catheter Cardiovasc Interv 2017;90.

23. Wong SC, Pawar S, Minutello RM, et al. Device success and 30-day clinical outcome in patients undergoing preimplant valvuloplasty in Transfemoral versus omitting valvuloplasty in transapical transcatheter aortic valve replacement. J Thorac Cardiovasc Surg 2015;150:1111-7.

24. Litzler P-Y, Borz B, Smail H, et al. Transapical aortic valve implantation in Rouen: four years' experience with the Edwards transcatheter prosthesis. Arch Cardiovasc Dis 2012;105:141-5.

25. Thomas M, Schymik G, Walther T, et al. One-Year outcomes of cohort 1 in the Edwards SAPIEN aortic bioprosthesis European outcome (source) registry: the European registry of transcatheter aortic valve implantation using the Edwards SAPIEN valve. Circulation 2011;124:425-33.

26. Aalaei-Andabili SH, Beaver TM, Bavry AA, et al. Outcomes of direct transcatheter aortic valve replacement without balloon aortic valvuloplasty using a new generation valve. Cardiovasc Revasc Med 2019. doi:10.1016/j.carrev.2019.01.020. [Epub ahead of print: 23 Jan 2019].

27. Bagur R, Kwok CS, Nombela-Franco L, et al. Transcatheter aortic valve implantation with or without preimplantation balloon aortic valvuloplasty: a systematic review and Meta-Analysis. J Am Heart Assoc 2016;5. 